Sādhanā Vol. 38, Part 4, August 2013, pp. 571-589. (C) Indian Academy of Sciences

\title{
An evolutionary approach for colour constancy based on gamut mapping constraint satisfaction
}

\author{
SHAHIN SABOONI and MOHSEN EBRAHIMI MOGHADDAM*
}

\author{
Electrical and Computer Engineering Department, Shahid Beheshti University; \\ G.C, 1983963113 Tehran, Iran \\ e-mail: S_Sabooni@mail.sbu.ac.ir; m_moghadam@sbu.ac.ir
}

MS received 12 July 2012; revised 18 November 2012; accepted 14 December 2012

\begin{abstract}
Many colour constancy algorithms have been proposed to achieve a good performance in this field. The gamut mapping algorithm is one of the most accurate and promising algorithms based on gamut assumption: illuminant can be estimated by comparing the colours distribution in the current image to acanonical gamut (i.e., a pre-learned distribution of colours). However, the gamut mapping algorithm is precise but it is time consuming. Therefore, some other methods such as GCIE (gamut constrained illuminant estimation) and CGM (cubical gamut mapping) have been proposed which work faster. However, the results of such methods are poor when the source light is not in the pre-defined ones. In this paper, we propose an evolutionary algorithm for colour constancy based on gamut mapping assumption. This approach overcomes the mentioned problem in other gamut-based methods. The proposed evolutionary method uses a simple chromosome structure together with simple operators such as mutation, selection, and reproduction. Two versions of the proposed methods have been presented here. The first one works on image pixels, while the second one tries on image derivative. The experiments were done on three different data sets that are used in literature and results were satisfactory. The results showed that the proposed method is much improved when compared to other related methods in most of the time especially in the case of real world images.
\end{abstract}

Keywords. Computer vision; colour; colour constancy; illuminant estimation; constraint satisfaction.

\section{Introduction}

All light sources with their features and characteristics involve in colour events and manipulate the colour measuring. However, human visual system is somehow able to recognize the colour of objects irrespective of light used to illuminate the objects: this ability is called Colour

*For correspondence 
constancy (Ebner 2007). In other words, the colour constancy is defined as perceiving the same colour of an object despite differences in illumination (Foster et al 2006; Delahunt \& Brainard 2004). Applying colour constancy improves the precision of many colour image processing applications such as object recognition (Gevers \& Smeulders 1999), image classification (Renno et al 2005), colour appearance models (Fairchild 2005) and colour image feature detection (Gevers 2006).

Since the colour constancy problem has been presented, many algorithms have been proposed to achieve a good performance in this field. A comprehensive review may be found in Hordley (2006). The colour constancy algorithms can be divided into two categories. First one includes those algorithms which work with simple computations on simple statistics information from images. White Patch Retinex (Land 1977), Gray World (Buchsbaum 1980), Shade of Gray (Finlayson \& Trezzi 2004), and Gray-edge (van de Weijer et al 2007) are some algorithms in this category. The second category contains algorithms that use complex information obtained from a prior processing on image. Calculating the gamut of possible reflectances in image under specific illuminant or applying learning methods on image information such as neural networks or genetic algorithms are examples of such processing. This category includes algorithms such as CRULE algorithm (Forsyth 1990), Colour-by-Correlation method using neural networks (Finlayson et al 2001; Cardei et al 2002), Ebner (2006) used algorithm genetic evolving approach and Bayesian colour constancy by Brainard \& Freeman (1997). Commonly these algorithms have good and sufficient results in colour constancy problem.

However, most proposed algorithms in colour constancy use several assumptions in their work (Akhavan \& Ebrahimi Moghaddam 2010, 2011), but all of them are on the foundation of certain assumption which suggested in the primitive works. For example, white-patch algorithm relies on having a white patch somewhere in image that reflects the maximum light possible which is called 'white-patch assumption' (Land 1977). Gray-Edge algorithm assumes that the average of differences in the scene is achromatic (van de Weijer et al 2007) as well as higher order statistics (Gijsenij et al 2010). CRULE as a gamut mapping algorithm which has been introduced in Forsyth (1990) uses this assumption that the illuminant can be estimated by comparing the distribution of colours in the current image to a pre-learned distribution of colours (called the canonical gamut). Gamut mapping assumption is one of the best hypotheses on which solid methods were proposed.

Recently, some other approaches were tried on combining different methods using fuzzy techniques or neural networks (Akhavan \& Ebrahimi Moghaddam 2011; Faghih \& Ebrahimi Moghaddam 2011). Also, neural networks have been employed in a recent technique to improve the results of gray edge algorithm (Faghih \& Ebrahimi Moghaddam 2011).

In this paper, we propose a computational algorithm for colour constancy. The proposed method is based on an evolutionary approach to search the illuminant space and find the best fit for estimated illumination. This approach overcomes the main and important GCIE (gamut constrained illuminant estimation) constraint that limits it to choose estimated illuminant via three pre-defined and restricted illuminants. The experimental results were satisfactory and proposed method worked well on several data sets which consist of real world images. The results were better than many related works.

The rest of paper has been organized as follows: in section 2, we briefly describe colour constancy basis and reflectance model to introduce the colour constancy modelling. In section 3, the CRULE algorithm and its important derivations such as "Colour in perspective" (Finlayson 1996) and Edge-based gamut mapping (Gijsenij et al 2007, 2010) have been described. In section 4, we have detailed on GCIE method. In section 5, the proposed method is presented. In section 6, the experimental results are reported and the proposed method is 
compared to gamut mapping algorithms and also to other colour constancy methods. Finally, a brief conclusion is provided in section 7 .

\section{Background}

The colour constancy is the process of transforming the input colour image that has been captured in unknown illuminant to a colour image which has been captured under canonical illuminant (often white). To model this process, at first the image formation and reflection model should be specified and in continuance the Diagonal Model that discusses transform of an image from one illuminant to another should be considered.

\subsection{Reflection model}

An image I for a Lambertian surface can be formed by descriptor of surface reflectance $\mathrm{R}(\mathrm{x}$, $\lambda$ ), the scene Illuminant spectral power distribution (PSD) $L(\lambda)$ and camera sensitivity function $S_{i}(\lambda)$, where $\lambda$ is wavelength and $\mathrm{x}$ is the position of the object patch that is projected on the sensor array as given below:

$$
\mathrm{I}_{\mathrm{i}}(x)=\int_{\lambda_{0}}^{\lambda_{1}} \mathrm{~L}(\lambda) \cdot \mathrm{R}(x, \lambda) \cdot \mathrm{S}_{\mathrm{i}}(\lambda) \cdot \mathrm{d} \lambda,
$$

where $\left[\lambda_{0} \lambda_{1}\right]$ is visible spectrum and $\mathrm{i}=[\mathrm{R}, \mathrm{G}, \mathrm{B}]$.

However, as it has been shown in Vora et al (1997a, b, c) (as three parts) in most of real CCD cameras some other processes are performed to smooth the sharpness of input signal and resulting refined signal of the sensor. For example:

- Sensor response is not always linear along the amount of energy and a nonlinear function $F_{i}$ should be taken into account to model the sensor output.

- Since cameras were made for grabbing images to be displayed in a monitor, sensor outputs are softened by gamma correction using an exponential factor $\gamma_{i}$.

- Also, for the white correction in colour cameras and other electronic signal amplifications, an amplifying factor, $\alpha_{\mathrm{i}}$, is multiplied to sensor output.

- The dark current, $\delta_{h}$, may also occur. This is caused by an o? set noise of sensors that detect some light in darkness.

So to create a realistic and accurate model, the previous equation may be rewritten as follows:

$$
\mathrm{I}_{\mathrm{i}}(x)=\alpha_{\mathrm{i}}\left[\mathrm{F}_{\mathrm{i}}\left(\int_{\lambda_{0}}^{\lambda_{1}} \mathrm{~L}(\lambda) \cdot \mathrm{R}(\mathrm{x}, \lambda) \cdot \mathrm{S}_{\mathrm{i}}(\lambda) \cdot \mathrm{d} \lambda\right)\right]^{\frac{1}{\gamma_{i}}}+\delta_{\mathrm{h}},
$$

where $\mathrm{i}=[\mathrm{R}, \mathrm{G}, \mathrm{B}]$.

But to work with this model, first we should remove all the sensor nonlinearities. Vora et al (1997a, b, c) proposed some proper approaches to calibrate colour devices. Also, Barnard \& Funt (1999) suggested calibration as a first step in any colour research. In the underlying idea of this process, the sensor response is estimated varying the exposure under which the picture is taken. This observation permits estimating of a linearized response without any prior information of the scene radiance and no special device. 
Without going into more details on this subject, from now, we suppose that linearized sensors are used in by following equation (1).

\subsection{Diagonal model}

Since, colour constancy methods try to transform all input image colours to colours in a reference light source; we need a transformation model which is often considered as chromatic adaption (Fairchild 2005). This transformation may be done by a diagonal mapping proposed by von Kries (1970) Model. However, more processes such as colour basis (Chong et al 2007) or applying spectral sharpening (Finlayson et al 1994) may be done.

By applying the diagonal mapping, the image taken under reference light source (usually called canonical illuminant) is computed as $\mathrm{I}^{\mathrm{c}}=$ D.I $\mathrm{I}^{\mathrm{u}}$, where $\mathrm{I}^{\mathrm{u}}$ is the image taken under unknown illuminant, $\mathrm{I}^{\mathrm{c}}$ is the image come into view under canonical illuminant and $\mathrm{D}$ is a diagonal matrix which maps the colours of input image to their corresponding colour under canonical illuminant $\mathrm{c}$ :

$$
\left[\begin{array}{l}
R^{c} \\
G^{c} \\
B^{c}
\end{array}\right]=\left[\begin{array}{lll}
\alpha & 0 & 0 \\
0 & \beta & 0 \\
0 & 0 & \gamma
\end{array}\right]\left[\begin{array}{l}
R^{u} \\
G^{u} \\
B^{u}
\end{array}\right] .
$$

However, in some cases, it is not possible to find such diagonal map; to overcome this problem Finlayson (1996) has recommended adding an offset to the diagonal model which is called offset diagonal model.

$$
\left[\begin{array}{l}
R^{c} \\
G^{c} \\
B^{c}
\end{array}\right]=\left[\begin{array}{lll}
\alpha & 0 & 0 \\
0 & \beta & 0 \\
0 & 0 & \gamma
\end{array}\right]\left[\begin{array}{l}
R^{u} \\
G^{u} \\
B^{u}
\end{array}\right]+\left[\begin{array}{c}
O_{r} \\
O_{g} \\
O_{b}
\end{array}\right] .
$$

Offset diagonal model is good enough for transformation modelling and it has been used here.

\section{Gamut mapping algorithms}

In the gamut mapping approaches, it is assumed that in the real world under each illumination only restricted set of colours can be observed which this restricted set is called gamut and the illuminant can be estimated by comparing the gamut of input image under unknown light source and gamut of canonical light source (often white). So the aim is to find a mapping $\mathrm{T}$ which when applied to these sensor responses (image pixels), produces the sensor responses that would be recorded under a canonical illuminant. To achieve this aim, the diagonal model and diagonal matrix $D \in \mathcal{D}_{3}(\mathbb{R})$ are considered to map the gamut of input image colours $\Gamma(I)$ to a gamut of canonical colours $\Gamma(\mathcal{C})$. The first step of the algorithm is to find the set of all feasible matrices D such that $\forall c \in \Gamma(I), D . c^{T} \in \Gamma(\mathcal{C})$, where $\mathrm{c}=[\mathrm{R}, \mathrm{G}, \mathrm{B}]$ is the sensor response.

\subsection{Canonical gamut $\Gamma(\mathcal{C})$}

Canonical gamut is defined as set of all physically realizable surface reflectance that can appear under canonical illuminant which often is white light source. This set is always approximated to a convex combination of the set $C=\left\{c_{i}^{C}\right\}, i=1, \ldots, n$ of all sensor responses to $n$ surfaces:

$$
\Gamma(C)=\left\{\sum_{i=1}^{n} w_{i} c_{i}^{c} \mid c_{i}^{c} \in C, w_{i} \geq 0 \forall i=1, \ldots, n \text { and } \sum_{i=1}^{n} w_{i}=1\right\} .
$$


Since $\Gamma(C)$ is a convex set, we can just consider the group of points defining the convex hull of $\mathrm{C}$ that is set $\mathcal{C}$. Therefore, $\Gamma(\mathcal{C})=\Gamma(C)$.

To compute the convex hulls, Finlayson et al (2005) introduced an efficient implementation using convex programming.

\subsection{Image gamut $\Gamma(\mathcal{J})$}

The image gamut is defined in a similar way from the set of image colours $I$ recorded under an unknown illuminant. Then, all convex combinations of I $(\Gamma(I))$ could be observed under this illuminant. Again, since the set of $\Gamma(I)$ is also a convex set; it makes possible the mere consideration of the convex hull $\Gamma(\mathcal{J})$ instead of $(\Gamma(I)$.

\subsection{Set of feasible mappings $\Gamma(\mathcal{C} / \mathcal{J})$}

As it said, a feasible solution could be a diagonal map D that maps a point in the image gamut somewhere in the canonical gamut. The key idea of this approach is to only using the points of the image and the canonical convex hull to compute the set of all feasible mappings.

For each convex hullvertex of the input image gamut, $c_{i}^{I} \in I$, a set of mapping that maps this point in the convex hull of the canonical gamut $\mathrm{C}$ can be computed by dividing each point in $\mathrm{C}$ by $c_{i}^{\mathrm{I}} \in \mathrm{I}$ component-wise.

Therefore, $\Gamma\left(\mathrm{C} / \mathrm{c}_{\mathrm{i}}^{\mathrm{I}}\right)$ is the set of all convex combination of the set $\mathrm{C} / \mathrm{c}_{\mathrm{i}}^{\mathrm{I}}$, where $\mathrm{C} / \mathrm{c}_{\mathrm{i}}^{\mathrm{I}}$ is:

$$
C / c_{i}^{I}=\left\{D^{j} \in D_{3}(\mathbb{R}) \mid D_{k}^{i}=c_{j, k}^{C} / c_{j, k}^{I}, \forall c_{j}^{C} \in C \text { and } 1 \leq k \leq 3\right\} .
$$

As it follows, the intersection of all these sets of mapping presents the set of feasible mappings:

$$
\Gamma(\mathrm{C} / \mathrm{I})=\cap_{\mathrm{i}=1}^{\mathrm{n}} \Gamma\left(\mathrm{C} / \mathrm{c}_{\mathrm{i}}^{\mathrm{I}}\right) .
$$

\subsection{Selection of one single mapping}

Once the feasible mapping set is found, it is time to select one single mapping from the whole set to yield a singular estimate of the unknown illuminant. Forsyth picked out a heuristic by which the volume of $\Gamma(\mathrm{I})$ mapped inside $\Gamma(\mathrm{C})$ is maximum (Forsyth 1990). Finlayson employed another heuristic to maximize the area of the canonic set occupied by the input image gamut according to diagonal mapping (Finlayson 1996; Finlayson et al 2001). Also, if we assume that all mapping and their corresponding illuminant are equal likely, the mean value of the mapping set is a good estimate (Barnard et al 2002a, b, c). Finlayson \& Hordley (1999) showed, this mean estimate provides better colour constancy results than the Forsyth's heuristic in general.

\subsection{Developing of gamut mapping algorithm}

Finlayson (1996) simplified gamut mapping algorithm and showed that it is possible to use all assumptions of $3 \mathrm{D}$ gamut mapping in a $2 \mathrm{D}$ space like chromaticity space $(R / B, R / G)$. Theoretically, these factors only affect the intensity of the recovered illuminant rather than its colour. Finlayson \& Hordley $(1999,2000)$ proved an important result: the set of 3D diagonal matrices recovered using the Forsyth's algorithm, when projected into a 2D chromaticity space is identical to the set of 2D diagonal matrices recovered by the Finlayson's algorithm using perspective colour. 
As a consequence, if the intensity of the scene illumination is not strictly necessary or recovered by an alternative way, it is far better to work in a $2 \mathrm{D}$ chromaticity space because computations are easier than 3D.

Recently Gijsenij et al (2007, 2010) have combined gray-edge assumption with the gamut mapping algorithm and proposed a new approach to gamut mapping algorithms. They introduced a framework which works on the local n-jet describing the derivative structure of an image (Kass \& Witkin 1987; Koenderink \& van Doorn 1987) and showed that the derivative-based approaches and also n-jet based gamut mapping have better performance where compared to the pixel-based gamut mapping and the fusion strategy based on the intersection of feasible sets provides better colour constancy results than the union of the feasible sets.

One of the accurate and important derivations of gamut mapping algorithm introduced by Finlayson is called GCIE (Finlayson et al 2006). In this paper, we took a special interest on GCIE to improve its flexibility and adaptation.

\subsection{Gamut constrained illuminant estimation}

There is a big problem in original gamut mapping algorithm: the intersection of all possible mappings for each vertex may be empty. Therefore, since the original work of Forsyth, many algorithms have been proposed to overcome this problem. GCIE is analgorithm which overcomes this problem successfully. In GCIE, it is assumed that the $i^{\text {th }}$ plausible illuminant is the scene illuminant, therefore, if this hypothesis is true, then all the image colours fall within the gamut for that light. Otherwise, some of the image colours will fall outside the gamut. In both cases, it is possible to measure the error in the hypothesis according to how far outside from the gamut the image colours are. After this hypothesis Finlayson proposed an error measure to determine consistency of each plausible illuminant with the image data. First, let us consider $\mathrm{G}=\left\{\mathrm{p}_{1}^{\mathrm{i}}, \mathrm{p}_{2}^{\mathrm{i}}, \ldots, \mathrm{p}_{\mathrm{N}}^{\mathrm{i}},\right\}$ as the set of sensor responses of a device to the $\mathrm{N}$ surfaces under the $i^{\text {th }}$ illuminant, Forsyth (1990) said that if $\mathrm{N}$ surfaces in $\mathrm{G}$ are observable under the $i^{\text {th }}$ illuminant, then all convex combinations of the elements of $\Gamma(\mathrm{G})-$ let $\Gamma(\mathrm{G})$ be convex hull of $\mathrm{G}-$ are observable under the $i^{\text {th }}$ illuminant:

$$
p_{k}^{i}=\sum_{p_{j}^{i} \in \Gamma(G)} \alpha_{j} p_{j}^{i}, \forall \alpha_{j} \geq 0, \sum \alpha_{j}=1
$$

Finlayson used above equation and introduced an error measure by finding $\alpha_{\mathrm{j}}$ which minimizes the $\mathrm{e}_{\mathrm{k}}^{\mathrm{i}}$ as follows:

$$
\begin{gathered}
e_{k}^{i}=\left\|p_{k}^{o}-\sum_{q_{j}^{i} \in \Gamma(G)} \alpha_{j} q_{j}^{i}\right\|^{2}, \\
e_{\text {total }}^{i}=\sum_{k=1^{n}} e_{k}^{i},
\end{gathered}
$$

where $\mathrm{e}_{\mathrm{k}}^{\mathrm{i}}$ is the error for $k^{\text {th }}$ surface (or $k^{\text {th }}$ vertex of gamut) and $\mathrm{e}_{\text {total }}^{\mathrm{i}}$ is the total error when the $i^{\text {th }}$ illuminant is considered as the scene light. Finding $\alpha_{\mathrm{j}}$ which minimizes the error $\mathrm{e}_{\mathrm{k}}^{\mathrm{i}}$ is a non-negative least squares problem.

In GCIE to test the illuminant as the scene light, Finlayson used three sets of illuminants; a set with 11 illuminants which represents the situation in which we have maximal prior knowledge about the scene illuminant, a set with 28 illuminants which includes a variety of the most commonly occurring lights, and another set which includes 87 illuminant measured by Barnard et al (2002a, b, c) and represents the case in which only limited prior knowledge about the scene illuminant is available. 
Via these three sets, Finlayson introduced three version of GCIE in which each of them has a very good accuracy: 11 lights version, 28 lights version, and 87 lights version.

Later, Mosny \& Funt (2010) proposed a new algorithm called CGM (Cubical Gamut Mapping) which in their work instead of computing the gamut of each image, they computed a bounding cube by simply determining the largest and smallest values of rgb chromaticity. This approach has simplified GCIE and decreased its error. The main advantages of CGM over GCIE are its simplicity and stability according to their results (better RMS and MAX angular error). So we can consider CGM as a simplified and stable version of GCIE.

One of the concerns of these two methods is that they are totally dependent on the set of lights. In other words, if an image exists that its source light is not in the considered illuminant set or there are more than one candidate in the sets, the results would be worse. The evidence of this concern occurred in the experimental results of GCIE: when the number of lights in the set increased, the method got worse results. Here, we try to overcome this concern by proposing an evolutionary approach in the next section.

\section{Proposed work}

As it was said in the previous section, GCIE reduced the problem of finding the plausible mapping to the problem of illuminant classification and reached to a solid performance (like what CGM does). A problem exists yet: Finlayson has tested his algorithm on a set that was captured under an indoor scene and with a set of artificial illuminants which makes the captured images differ from real-world images. Another important weakness of GCIE (which occurred also in CGM) is that the illuminant which is used for train phase is actually the illuminant have been used in Barnard's data set (Barnard et al 2002c). Barnard used 11 lights to make his data set and these lights are same lights which used in 11-lights version of GCIE. In other versions of GCIE, these 11 lights or some others close to them were also used.

It seems the results achieved by Finlayson are not defendable in real-world conditions (because in real world, we have more illuminants - as we can see in Ciurea \& Funt (2004) real-world image data set).

In this situation, to overcome the deficiency of confined illuminant sets of GCIE algorithm, we could have a procedure which chooses a target illuminant from huge dynamic illuminants set. In an overall view, we could model this objective procedure as a searching problem. In our work, we used an evolutionary algorithm to search via a huge dynamic set of illuminants and find the best estimated consistent illuminant.

Importance of using an evolutionary algorithm for this problem would be illustrated by considering this note about the problem. The search space has $256^{3}=16,777,216$ points. If we have to search this space by a brute force method and if measuring test for each point takes 0.5 second, we need $8,388,608$ seconds $(\approx 139,810$ minutes $\approx 2,330$ hours $\approx 97$ days) to complete search and reach the optimal result. As it is seen, computing the best result is time consuming and it is not efficient for colour constancy work. Even Ebner (2006) evolutionary method which takes about 10 days and has a good result isnot noticeable for many colour constancy researchers. So, we propose a method to perform this search in short time and reach solid results. Since one of the evolutionary algorithm phases is mutation, selecting an evolutionary method gives us the advantage of searching the entire search space without going straight to local minima. This advantage is more important in colour constancy by considering that most of colour constancy methods use a certain assumption which is not valid in all conditions and it is very good to look around the assumed best point for real better points. 
Before we describe the evolutionary method, the pre-processing phase of proposed method should be considered.

\subsection{Pre-processing}

To prepare the input image for proposed algorithm, some pre-processing techniques employed as follows:

4.1a Quantizing RGB: In this pre-processing phase, each colour channel was quantized into 128 intervals resulting in a total of 2,097,152 bins. The average RGB value from each interval is used as the pre-processing output. This phase has been employed to remove zero values to avoid division by zero errors in the proposed approach.

4.1b Dark Pixel Removal: Pixels with $\mathrm{R}+\mathrm{G}+\mathrm{B}<T h r$ are removed. As well, brighter pixels have a higher chance of being directly illuminated by the main illuminant. In some other works like Hordley \& Finlayson (2006). Dark pixel removal was used also. In the proposed approach, the $T h r$ value 'is considered as 10 percent of max value (which is 256).

4.1c Even Blocks: This pre-processor introduced in Mosny \& Funt (2010) work. Each pixel is considered relative to its $\mathrm{N} \times \mathrm{N}$ neighborhood of surrounding pixels. If the $\mathrm{R}, \mathrm{G}, \mathrm{B}$ (or $\mathrm{rg}$ chromaticity) values of each surrounding pixel do not differ from means of the R, G, B (or rg chromaticity) values in the neighborhood by more than some threshold then the central pixel is kept and the pre-processing output is set to be the mean value of the pixels in the neighborhood. If any one of the R, G, B (or rg chromaticity) values from any of the surrounding pixels differs from the R, G, B (or rg chromaticity) means by more than the given threshold, then the central pixel is flagged as unreliable in the output. Finally we can simply disregard all such flagged pixels (Mosny \& Funt 2010). In our algorithm, threshold is 10 percent of max value and $\mathrm{N}=5$. As it has been shown in Mosny \& Funt (2010) this pre-processing performs better than Gaussian smoothing (van de Weijer et al 2007; Gijsenij et al 2010) and block averaging (Barnard et al $2002 \mathrm{a}, \mathrm{b}, \mathrm{c})$. Also this preprocessor is employed to smoothen the convex hull of gamut and decreases the gamut mapping complexity.

\subsection{Evolutionary algorithm}

We propose an evolutionary algorithm to search via a huge dynamic set of illuminants and find the best estimated consistent illuminant. This procedure is as follows: first, to make a startup generation 100 lights are selected uniformly (as RGB value) from colour spectra of black-body radiator (Burnham et al 1963). It is because the natural illuminant (which can be obtained via black-body radiator) can be used to constrain the infinitely large set of possible illuminants. Ebner (2007, pp. 126-127) which uses black-body radiator as a light source to approximate the natural light sources.

Since in this approach (searching for the plausible light whose gamut is most consistent with the image data) good gamut is the optimally restricted gamut and not a big and wide shape one (Finlayson et al 2006), we should start with dark illuminant and then in searching process brighten them by and by. Therefore, to make the initial population darker, the RGB value of illuminant is divided by 2.5 and its interval is mapped from [0 255] to [0 100]. 


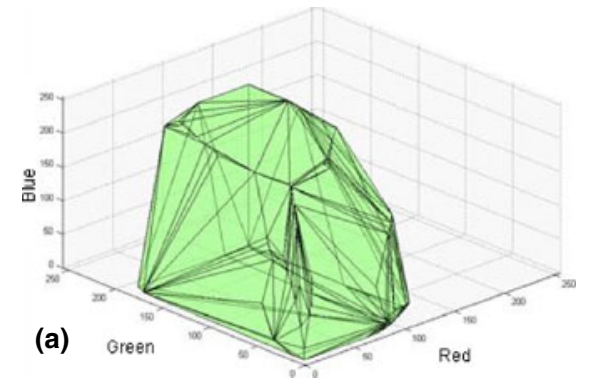

$\operatorname{Light}\left[\begin{array}{l}R \\ G \\ B\end{array}\right]=\left[\begin{array}{l}103.5 \\ 167.3 \\ 226.3\end{array}\right]$

(b)

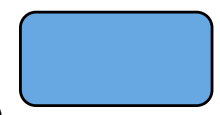

Figure 1. Visualization of one chromosome. (a) Visualization of illuminant gamut; (b) light and it's colour.

Each chromosome in the evolutionary algorithm is considered as an illuminant ([R, G, B]). In the evolution process, for each chromosome its corresponding gamut is extracted by the presented method in Gijsenij et al (2010). In figure 1, a sample chromosome structure with its visual representation has been shown.

As the fitness function in the proposed algorithm, the error measure function (equation 10) which has been proposed in Finlayson et al (2006) is used. The reasons for selecting equation (10) as fitness are as follows: first, proposed measure function is based on gamut mapping assumption so we can test hypothesized illuminant as scene illuminant and see all the observed gamut points fall into illuminant gamut or not, and if it is not, measure the error of hypothesized illuminant consistency. Existence of a simple and fast solution (non-negative least squares algorithm) for this, error measure function is the second reason. But this error measure has a disadvantage beside its benefits. It can become zero for some illuminant which has a large gamut so the searching method stops while we are not close to the desired answer. To avoid or decrease this problem, the search is started with dark illuminants (which have a small gamut) and illuminants are brightenedby and by. Also they are not allowed to brighten so much at once. This procedure will be explained further more.

After measuring fitness for each population, we have to:

(i) Select some of top results and send them to the next generation. For this stage, we consider selecting 5 percent of best chromosomes.

(ii) Manipulate some of them and make a new test light from search space. For manipulating, we change illuminant tristimulus ( $\mathrm{R}, \mathrm{G}, \mathrm{B})$ values by adding or subtracting to/from a random value. In the proposed approach, to have a non-blind work, first the principal components of illuminants gamut and input images gamut are measured, then using these measures the illuminant tristimulus values are manipulated as follows:

$$
\left[\begin{array}{c}
R_{i}^{\text {new }} \\
G_{i}^{\text {new }} \\
B_{i}^{\text {new }}
\end{array}\right]=\left[\begin{array}{c}
R_{i} \\
G_{i} \\
B_{i}
\end{array}\right]+W \cdot\left[\begin{array}{c}
\delta R_{i} \\
\delta G_{i} \\
\delta B_{i}
\end{array}\right] \odot\left[\begin{array}{c}
M a x_{c h}-R_{i} \\
M a x_{c h}-G_{i} \\
M a x_{c h}-B_{i}
\end{array}\right],
$$

where matrix $\delta \mathrm{s}$ is difference of illuminant gamut axel RGB values from input image gamut axel RGB values, $M_{a x}$ is maximum of tristimulus channels which in this work is 256 and $\mathrm{W}$ is increasing factor for brightening illuminant by and by. The value of $\mathrm{W}$ has been considered as 0.2 experimentally. In fact the last matrix of above equation is employed to change small values by more amount of change than larger values. 
By above equation, for example, if we see a more amount of red value in the axel of input image gamut than axel of illuminant gamut, we should add bigger value to illuminant tristimulus values.In the proposed method, $70 \%$ of population is manipulated.

(iii) Mutate some of chromosomes for considering the whole search space. As it said, we begin with dark illuminant and in search process we brighten illuminant to search all possible illuminant without being captured by a wide gamut which has an unexpected illuminant. So in the mutation stage, the illuminant is brightened via increasing intensity value of illuminant. We mutate $2 / 7$ of manipulated chromosome (20\% of population) and $15 \%$ of population in this stage.

Finally to complete the population again the $10 \%$ remaining population are reproduced by regeneration.

The termination condition of evolutionary approach is considered as reaching to zero in fitness or doing 100 iterations.

In figure 2, the proposed method is shown. Figure 3 shows the mechanism of the proposed method in some diagrams. In figure 3 a you can see the input image for testing that is selected from presented database in (Ciurea \& Funt 2004). This test takes 44 iterations to complete. The evaluation of fitness function in 44 iterations has been shown in figure $3 \mathrm{~b}$. Also, in figure $3 \mathrm{c}$ the best estimated illuminant in each iteration (red pluses), target illuminant (blue circle), and span of all tested illuminant in this run (gray area) has been illustrated.

In another version of the proposed method (version 2), we employed gray-edge assumption and recent work of Gijsenij et al (2010). Gijsenij et al showed that derivative structure of an image has the advantage over pixel values to be invariant to disturbing effects (i.e., deviations of the diagonal model) such as saturated colours and diffuse light. They also showed that the n-jet completely describes the derivative structure of an image. Hence, instead of using pixel values only, edges and higher-order structures are included in the $n$-jet description,and the gamuts of the $n$-jet behave similarly under illuminant variations as a normal zero-order gamut. Therefore, in version 2, we used 1-jet description in the same way as Gijsenij et al (2010) to compute fitness function.

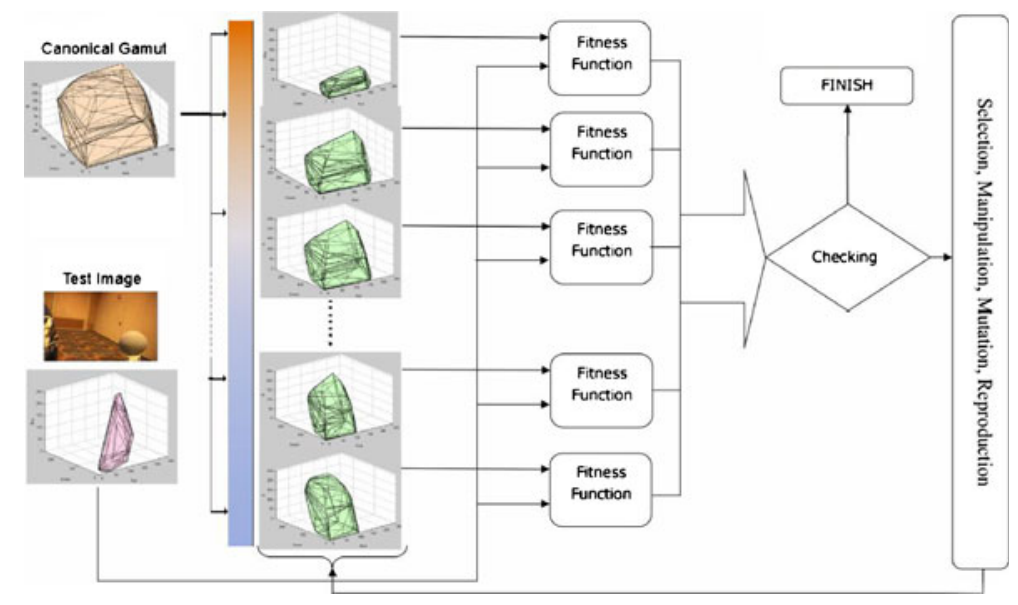

Figure 2. Diagram of the proposed algorithm. 


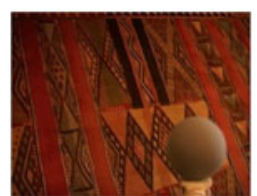

(a) input Image for Testing

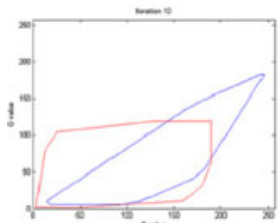

(e) RG plane of gamuts after 10 iterations

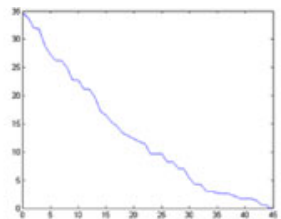

(b) fitness evaluation over 44 iterations

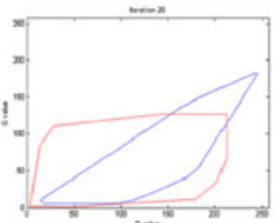

(f) RG plane of gamuts after 20 iterations

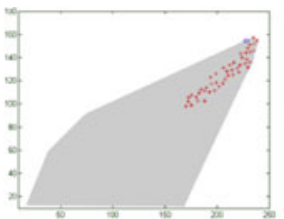

(c) Best estimated illuminant in each iteration (red), target illuminant (blue) and span of all tested illuminants (gray area)

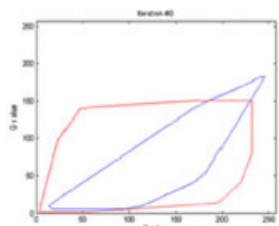

(g) RG plane of gamuts after 30 iterations

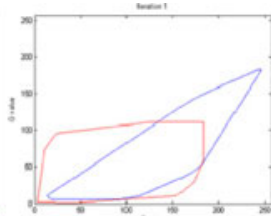

(d) RG plane of gamuts after first iteration, Illuminant gamut (red), Input image gamut(blue)

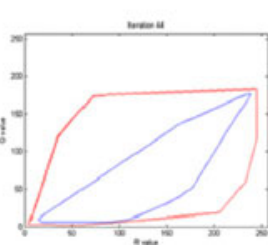

(h) RG plane of gamuts after 44 iterations

Figure 3. Mechanism of proposed method in some diagrams.

After determining the methods, some experiments were done with the proposed methods to find out their performance.

\section{Experiments}

To evaluate the performance of the proposed method, several datasets were employed and results were reported in terms of angular error which is an intensity-independent measure as follows:

$$
\mathrm{e}_{\text {ang }}=\cos ^{-1}\left(\frac{\widehat{\mathrm{p}} \cdot \mathrm{p}}{\|\widehat{\mathrm{p}}\| \cdot\|\mathrm{p}\|}\right),
$$

where $\widehat{p}$ is the estimated RGB and $\mathrm{p}$ is the actual RGB of illuminant.

The angular error was selected as a performance measure evaluation, since it has been shown in other researches that it is a good measure to compute performance of colour constancy methods and also the median angular error is reported as summarizing statistic (Hordley \& Finlayson 2006; Gijsenij et al 2009).

To evaluate both versions of the proposed method, we chose three different data sets which have been used for some previous works. These data sets were: Barnard et al (2002a, b, c) data set which has been captured under laboratory conditions, Ciurea \& Funt (2004) data set which has been taken from real-world scene and Gehler et al (2008) data set which contains image from different scenes and different conditions.

\subsection{Data set in laboratory conditions (Barnard et al 2002c)}

This experiment performed on real images taken under laboratory settings. All images in this data set are indoor scenes. There are 31 scenes, taken under 11 different light sources, resulting in a total of 321 images. The canonical gamuts for this dataset were computed by similar way as Gijsenij et al (2010).

Table 1 shows the performance of both versions of presented method in terms of median, mean, RMS, and maximum angular error using this data set. 
Table 1. The performance of both versions of proposed method using Barnard's data set (Barnard et al 2002c).

\begin{tabular}{lcr}
\hline & Version 1 & Version 2 \\
\hline Median & 2.74 & 2.17 \\
Mean & 3.73 & 3.34 \\
RMS & 5.13 & 5.11 \\
Max & 16.31 & 15.72 \\
\hline
\end{tabular}

\subsection{Real-world image data set (Ciurea \& Funt 2004)}

Next experiment performed on a set of real-world images. This set consists of images that are captured using a camera with a grey sphere mounted in front. There are 15 different scenes, indoor and outdoor conditions, resulting in a total of 11346 images. Also, the canonical gamuts were computed by taking one randomly selected image from each scene as mentioned in section 5.1.

The performance of both proposed versions in terms of median, mean, RMS and maximum angular error using this data set are shown in table 2.

\subsection{Gehlers image data set}

Third experiment performed on a set provided by Gehler et al (2008). The Gehler's dataset contains 568 images and includes a variety of indoor and outdoor shots taken using two high quality DSLR cameras (Canon 5D and Canon1D) with all settings in auto mode. Each image contains a MacBeth colourchecker for reference. In this experiment, we used an extended version of this data set provided by Chakrabarti et al (2010) in their work. In this new dataset, the dataset has grown to include about sixty new images of indoor scenes without daylight illumination. To make the canonical gamut from this data set, we used 14 images from different indoor and outdoor scene.

The performance of two versions of the proposed method in terms of median, mean, RMS and maximum angular error using this data set are shown in table 3.

\subsection{Time complexity and consuming time}

Time complexity of pre-processing phase is $\mathrm{O}(m \times n)$ where $m$ and $n$ are width and height of image in pixel respectively. In version 2, we calculate 1-jet descriptor before and after preprocessing phase. This phase is also computed in $\mathrm{O}(m \times n)$. At each iteration of evolutionary

Table 2. The performance of both versions of proposed method using real-world data set (Ciurea \& Funt 2004).

\begin{tabular}{lcr}
\hline & Version 1 & Version 2 \\
\hline Median & 2.28 & 2.02 \\
Mean & 3.35 & 3.14 \\
RMS & 5.06 & 5.01 \\
Max & 13.12 & 14.78 \\
\hline
\end{tabular}


Table 3. The performance of both versions of proposed method using Gehler data set.

\begin{tabular}{lcc}
\hline & Version 1 & Version 2 \\
\hline Median & 2.36 & 2.13 \\
Mean & 3.75 & 3.29 \\
RMS & 5.13 & 5.23 \\
Max & 17.22 & 17.81 \\
\hline
\end{tabular}

Table 4. Time consuming of both versions of proposed method for 100 image with resolution of $814 * 520$ on a PC with Intel $2.0 \mathrm{GHz}$ Dual Core2 CPU.

\begin{tabular}{lrr}
\hline & Version 1 & Version 2 \\
\hline Median & $93.1 \mathrm{~s}$ & $81.4 \mathrm{~s}$ \\
Mean & $104.8 \mathrm{~s}$ & $102.8 \mathrm{~s}$ \\
RMS & $67.7 \mathrm{~s}$ & $65.0 \mathrm{~s}$ \\
Max & $283.6 \mathrm{~s}$ & $275.7 \mathrm{~s}$ \\
\hline
\end{tabular}

search manipulating the population take a little time with complexity of $100 \times \mathrm{O}(1)$, but to calculate fitness function for each chromosome we have to solve a non-negative least square problem which is $\mathrm{O}\left(k^{3}\right)$, where $k$ is the number of points representing the convex hull of each chromosome. Finally, the time complexity of our proposed method is

$$
3 \times \mathrm{O}(m \times n)+100 \times\left[100 \times \mathrm{O}(1)+100 \times \mathrm{O}\left(k^{3}\right)\right]=O\left(m n+k^{3}\right) .
$$

Mean, median, RMS and maximum of time consuming of proposed algorithm version 1 and version 2 for 100 images with resolution of $814 * 520$ (resized almost from Gehler's dataset) on a PC with Intel 2.0 GHz Dual Core 2 CPU is reported in table 4. It is worth mentioning that we have not compared the absolute elapsed time of the proposed method with other gamut-based approaches, because the search space of different methods are different and this comparison is not fare. For example, GCIE method searches only in a finite set, so it converges so fast, but as we mentioned before, it works only for a finite set of lights.

\section{Comparisons}

To compare the proposed work with related ones, we chose two data sets which are the main data set for test in other colour constancy algorithms. Barnard's data set of laboratory captured images (Barnard et al data set 2002c) and real-world image data set (Ciurea \& Funt 2004). We extracted the result of other colour constancy algorithm from corresponding publications (van de Weijer et al 2007; Finlayson et al 2001; Gijsenij et al 2010; Finlayson et al 2006; Mosny \& Funt 2010).

In the first part of this section, the performance of proposed work is compared with gamut mapping algorithms and in the second part, we have shown the performance of method in comparison with more famous works in colour constancy. 
Table 5. Comparing proposed methods to gamut mapping algorithms using Barnard's laboratory data set.

\begin{tabular}{lcccc}
\hline & Median & Mean & RMS & Max \\
\hline Regular GM & 3.13 & 5.7 & 7.2 & 26.13 \\
Regular GM (offset-model) & 2.92 & 4.17 & 5.6 & 23.19 \\
n-jet-based GM & 2.1 & 4.2 & - & - \\
Proposed method ver.1 & 2.36 & 3.73 & 5.13 & 16.31 \\
Proposed method ver.2 & 2.17 & $\mathbf{3 . 3 4}$ & $\mathbf{5 . 1 1}$ & $\mathbf{1 5 . 7 2}$ \\
Cubical GM & 2.46 & 3.77 & 5.22 & 19.34 \\
GCIE 11-lights & $\mathbf{1 . 3 1}$ & 4.18 & 6.88 & 27.64 \\
GCIE 28-lights & 2.14 & 4.3 & 6.95 & 27.64 \\
GCIE 87-lights & 2.6 & 4.75 & 7.11 & 19.43 \\
\hline
\end{tabular}

\subsection{Comparison with other gamut mapping algorithms}

In this part, the performance of the proposed work is compared with other gamut mapping algorithms. We will explain all advantages and disadvantages of the proposed method here.

Table 5 shows the result of gamut mapping algorithms using Barnard's data set of laboratory. Also, the results achieved by gamut mapping algorithms using real-world data set (Ciurea \& Funt 2004) are shown in table 6.

As you see in table 5, the best median angular error has been obtained by GCIE 11-light version. But as mentioned before in section 3.6, GCIE has been trained by the 11 illuminant which is used by Barnard to make his data set and as Finlayson said (Finlayson et al 2006), this version represents the situation in which we have maximal prior knowledge about the scene illuminant.

Table 6. Comparing proposed methods to gamut mapping algorithms using real-world image data set.

\begin{tabular}{lcc}
\hline & Median & Mean \\
\hline Regular GM (offset-model) & 5.5 & 7.2 \\
$n$-jet -based GM & 4.8 & 6.5 \\
Proposed method ver.1 & 2.28 & 3.35 \\
Proposed method ver.2 & $\mathbf{2 . 0 2}$ & $\mathbf{3 . 1 4}$ \\
\hline
\end{tabular}

Table 7. Comparing proposed methods to gamut mapping algorithms using Barnard's image data set (Ciurea \& Funt 2004).

\begin{tabular}{lcc}
\hline & Median & Mean \\
\hline White patch & 6.5 & 7.22 \\
Gray-world & 6.95 & 7.8 \\
Shade of Gray & 3.9 & 6.3 \\
Gray-edge & 3.2 & 3.9 \\
Gray-edge2nd-order & 2.7 & 3.8 \\
Proposed method ver.1 & 2.36 & 3.73 \\
Proposed method ver.2 & $\mathbf{2 . 1 7}$ & $\mathbf{3 . 3 4}$ \\
\hline
\end{tabular}




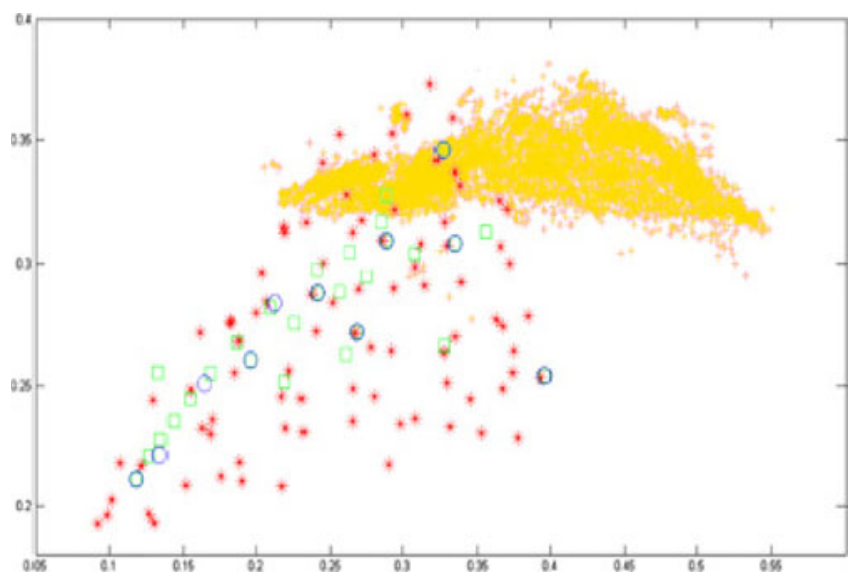

Figure 4. Representation of illuminant which used in GCIE and which used in Ciurea \& Funt (2004) real-world data set. In this figure, 11-light version (blue circles), 28-light version (green squares), 87-light version (red asterisks) and real-world illuminants (yellow points) in rg-chromaticity are shown.

So, as we can see in some other work in colour constancy, it is not necessary to compare the proposed work with GCIE, if we do not use maximal prior knowledge about the scene illuminant. Also, GCIE 28-light version uses 28 lights so close to 11-light version. However, the proposed algorithms have acceptable result in median angular error and show a good and stable functionality to other gamut mapping algorithms. Also, the proposed approach has resulted 15.72 in maximum angular error where as other gamut mapping algorithms has 19.34.

In real-world images unfortunately we could not find any results for GCIE versions or CGM; however it seems that these algorithms cannot yield acceptable results, because, as it is obvious in figure 4 all used illuminants for these algorithms have large difference to illuminants with illuminate Ciurea \& Funt (2004) real-world data set scenes.

By the way, we can see improvement more than $55 \%$ in median and more than $45 \%$ in mean angular error with regard to other methods.

\subsection{Comparison to other colour constancy algorithms}

In this part, the performance of the proposed works is compared to some other works in colour constancy. We have explained all the advantages and disadvantages of the proposed method here.

Table 8. Comparing proposed methods to gamut mapping algorithms using real-world image data set (Ciurea \& Funt 2004).

\begin{tabular}{lcc}
\hline & Median & Mean \\
\hline White patch & 6.7 & 7.1 \\
Gray-world & 7 & 7.9 \\
Gray-edge & 4.1 & 5.2 \\
Gray-edge2nd-order & 4.3 & 5.6 \\
NIS (Gijsenij \& Gevers 2011) & 3.7 & - \\
Proposed method ver.1 & 2.28 & 3.35 \\
Proposed method ver.2 & $\mathbf{2 . 0 2}$ & $\mathbf{3 . 1 4}$ \\
\hline
\end{tabular}


Tables 7 and 8 show the results achieved by the proposed methods in comparison to other colour constancy algorithms using Barnard's laboratory settings data set (Barnard et al 2002a, b, c) and real-world image data set (Ciurea \& Funt 2004), respectively.

In laboratory settings we had good promising algorithms like gray-edge and gray-edge $2^{\text {nd }}$ order, however as it is shown in table 6 the proposed methods outperform these algorithms by up to $18 \%$ improvement.

In real-world data set (Ciurea \& Funt 2004), as you see in table 8, the proposed method outperforms NIS method (Gijsenij \& Gevers 2011) up to $45 \%$ and by this comparison it is clearly
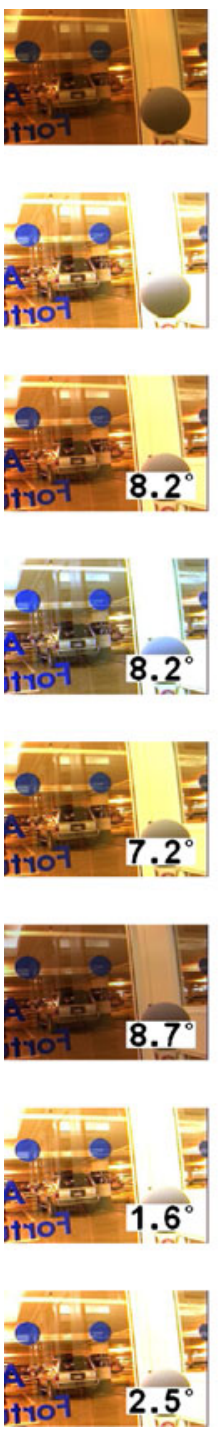
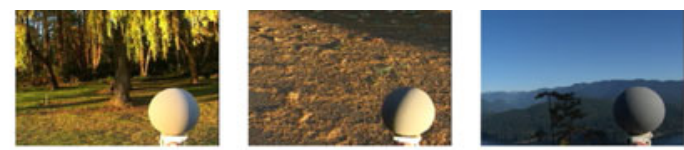

(a) Original Test Images
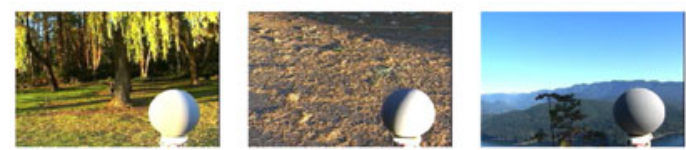

(b) Ideal Corrected Images

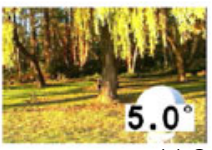

(c) Corrected by Max-RGB Method

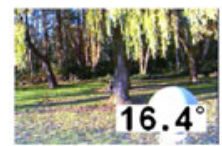

(c) Corrected by Gray World Method
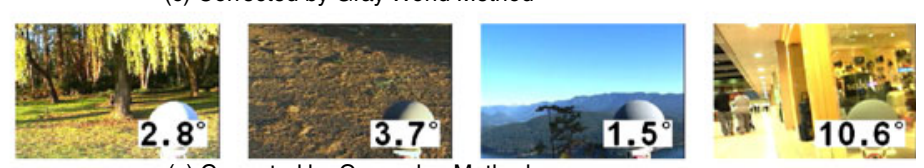

(c) Corrected by Gray-edge Method
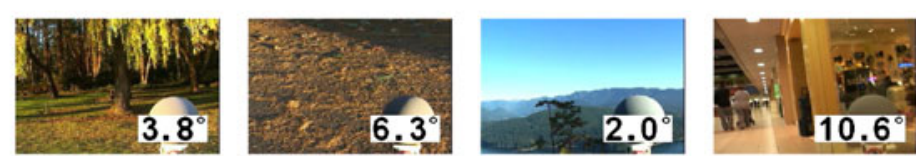

(c) Corrected by 1 -jet Intersection Gamut Mapping Method
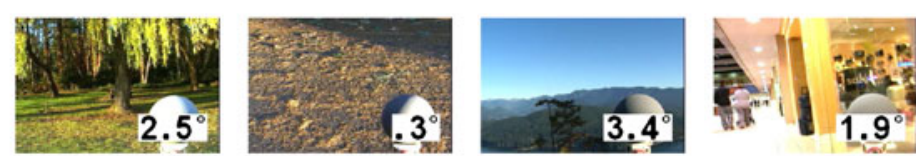

(c) Corrected by Proposed Method ver.1
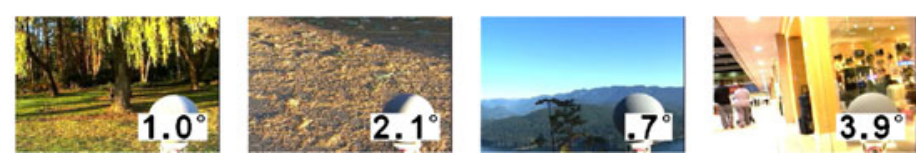

(c) Corrected by Proposed Method ver.2
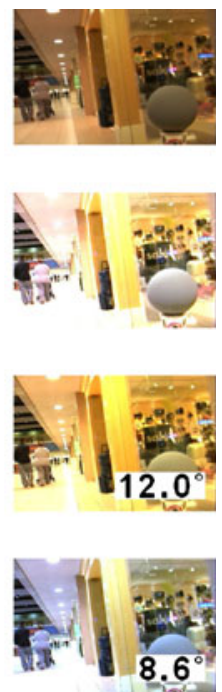

Figure 5. Examples of images in real-world data set (Ciurea \& Funt 2004) and results of some colour constancy algorithms. The corresponding angular errors are shown in the lower right corner of the images. 
shown that the proposed approach can be a strong, stable and promising algorithm for colour constancy especially in real-world conditions.

Figure 5 shows examples of images in real-world data set (Ciurea \& Funt 2004) and corresponding ideal colour correction and corresponding results of white-patch, gray world, gray-edge, gamut mapping using 1-jet intersection (intersection of $\mathrm{f}_{\mathrm{x}}$ and $\mathrm{f}_{\mathrm{y}}$ ) and the proposed methods. The angular error of each output is shown in the lower right corner of the images.

\section{Conclusion}

In this paper, we have proposed a new evolutionary approach for colour constancy problem. In the proposed method, we have tried to find a best fit of illuminant gamuts for satisfying gamut mapping constraint. The proposed method approach does not use any extra information and does not take too much time to find the result. As it has been shown in experiments section, the proposed method is so solid and stable in different conditions. In comparison section, it has been shown that the proposed approach outperformed gamut mapping algorithms up to $55 \%$ and also surpassed other colour constancy algorithms in laboratory settings up to $18 \%$ and in real-world scenes up to $45 \%$.

\section{References}

Akhavan T and Ebrahimi Moghaddam M 2010 A new combining learning method for colour constancy. In: 2nd International Conference on Image Processing Theory, Tools and Applications, p. 421-425

Akhavan T and Ebrahimi Moghaddam M 2011 A colour constancy method using fuzzy measures and integrals. Opt. Rev. 18(3): 273-283

Barnard K and Funt B V 1999 Camera calibration for colour vision research. In: SPIE Conference on Electronic Imaging Proc. Human Vision and Electronic Imaging IV, volume 3644, p. 576-585

Barnard K, Cardei V C and Funt B 2002a A comparison of computational colour constancy algorithms; part i: Methodology and experiments with synthesized data. IEEE Trans. Image Process. 11(9): 972-984

Barnard K, Martin L, Coath A and Funt B 2002b A comparison of computational colour constancy algorithms; part ii: Experiments with image data. IEEE Trans. Image Process. 11(9): 985-996

Barnard K, Martin L, Funt B and Coath A 2002c A data set for colour research. Colour Res. Appl. 27(3): $147-151$

Brainard D H and Freeman W T 1997 Bayesian colour constancy. J. Opt. Soc. Am. 14: 1393-1411

Buchsbaum G 1980 A spatial processor model for object colour perception. J. Franklin Inst. 310(1): 1-26

Burnham R W, Haynes R M and Bartleson C J 1963 Colour: A Guide to Basic Facts and Concepts. New York: John Wiley \& Sons

Cardei V C, Funt B and Barnard K 2002 Estimating the scene illuminant chromaticity by using a neural network. J. Opt. Soc. Am. 19(12): 2374-2386

Chakrabarti A, Hirakawa K and Zickler T 2010 Computational Colour Constancy with Spatial Correlations. Tech. Rep. TR-09-10, Harvard Sch. of Engg. \& App. Available at www.eecs.harvard.edu/ $\sim$ ayanc/color-constancy/dbs.html

Chong H Y, Gortler S J and Zickler T 2007 The von Kries hypothesis and a basis for colour constancy. In: Proc. of the International Conference on Computer Vision, p. 1-8

Ciurea F and Funt B 2004 A large image database for colour constancy research. In: Proc. IS\&T/SID's Colour Imaging Conf., Scottsdale, AZ, p. 160-64

Delahunt P B and Brainard D H 2004 Does human colour constancy incorporate the statistical regularity of natural daylight? J. Vis. 4(2): 57-81

Ebner M 2006 Evolving colour constancy. Pattern Recogn. Lett. 27(11): 1220-1229 
Ebner M 2007 Colour constancy. Wiley-IS\&T Series in Imaging Science and Technology. John Wiley \& Sons, Chichester

Faghih M and Ebrahimi Moghaddam M 2011 Neural gray edge: Improving gray edge algorithm using neural network. ICIP (International Conference on Image Processing) Proceeding, pp 1705-1708

Fairchild M D 2005 Colour appearance models (2nd ed.), Wiley-IS\&T series in imaging science and technology. John Wiley \& Sons, Chichester

Finlayson G D 1996 Colour in perspective. IEEE Transactions on Pattern Analysis and Machine Intelligence 18(10): 1034-1038

Finlayson G D and Hordley S D 1999 Selection for gamut mapping colour constancy. Image Vis. Comput. 17: 597-604

Finlayson G D and Hordley S D 2000 Improving gamut mapping colour constancy. IEEE Trans. Image Process. 9(10): 1774-1783

Finlayson G D and Trezzi E 2004 Shades of gray and colour constancy. In: Proc. IS\&T/SID Colour Imaging Conference, p. 37-41

Finlayson G D, Drew M S and Funt B V 1994 Spectral sharpening: sensor transformations for improved colour constancy. J. Opt. Soc. Am. A 11(5): 1553-1563

Finlayson G D, Hordley S D and Hubel P 2001 Colour by correlation: A simple, unifying framework of colour constancy. IEEE Trans. Pattern. Anal. Mach. Intel. 23(11): 1209-1221

Finlayson G D, Hordley S D and Xu R 2005 Convex programming colour constancy with a diagonal-offset model. In: Proceedings of IEEE International Conference on Image Processing, p. 948-951

Finlayson G D, Hordley S D and Tastl I 2006 Gamut constrained illuminant estimation. Int. J. Comput. Vis. 67(1): 93-109

Forsyth D 1990 A novel algorithm for colour constancy. Int. J. Comput. Vis. 5: 5-36

Foster D H, Amano K and Nascimento S M C 2006 Colour constancy in natural scenes explained by global image statistics. Vis. Neurosci. 23(3-4): 341-349

Gehler P, Rother C, Blake A, Minka T and Sharp T 2008 Bayesian colour constancy revisited. In: Proceedings of the IEEE Conference on Computer Vision and Pattern Recognition. At: http://www.kyb.mpg.de/bs/people/pgehler/colour/index.html

Gevers T 2006 Colour feature detection. An Overview Colour Image Processing: Methods and Applications, CRC Press

Gevers T and Smeulders A 1999 Colour based object recognition. Pattern Recog. 32: 453-464

Gijsenij A and Gevers T 2011 Colour constancy using natural image statistics and scene semantics. IEEE Trans. Pattern Anal. Mach. Intell. 33(4): 687-698

Gijsenij A, Gevers T and van de Weijer J 2007 Colour constancy by derivative-based gamut mapping. In: Photometric Analysis for Computer Vision (PACV'07), in Conjunction with ICCV'07, Rio de Janeiro, Brazil

Gijsenij A, Gevers T and Lucassen M 2009 A perceptual analysis of distance measures for colour constancy algorithms. J. Opt. Soc. Am. A 26(10): 2243-2256

Gijsenij A, Gevers T and van de Weijer J 2010 Generalized gamut mapping using image derivative structures for colour constancy. Int. J. Comput. Vis. 86(2-3): 127-139

Hordley S D 2006 Scene illuminant estimation: past, present, and future. Colour. Res. Appl. 31(4): 303-314

Hordley S D and Finlayson G D 2006 Reevaluation of colour constancy algorithm performance. J. Opt. Soc. Am. A 23(5): 1008

Kass M and Witkin A 1987 Analyzing oriented patterns. Comput. Vis., Graph. Image Process. 37(3): 362385

Koenderink J J and van Doorn A J 1987 Representation of local geometry in the visual system. Biol. Cybern. 55(6): 367-375

Land E H 1977 The retinex theory of colour vision. Scientific Am. 237(6): 108-128

Mosny M and Funt B 2010 Cubical Gamut Mapping Colour Constancy. In: Proc. CGIV2010 IS\&T Fifth European Conf. on Colour in Graphics, Imaging and Vision, Joensuu 
Renno J P, Makris D, Ellis T and Jones G 2005 Application and evaluation of colour constancy in visual surveillance. Presented at the Joint IEEE Int. Workshop Visual Surveillance and Performance Evaluation, Bejing, China

van de Weijer J, Gevers T and Gijsenij A 2007 Edge-based colour constancy. IEEE Trans. Image Process. 16(9): 2207-2214

von Kries J 1970 Influence of adaptation on the effects produced by luminous stimuli. In: Sources of Colour Vision, D. MacAdam (Ed.), MIT Press, Cambridge, p. 109-119

Vora P L, Farrell J E, Tietz J D and Brainard D H 1997a Digital colour cameras - 1 -response models. Technical Report HP-97-53, Hewlett-Packard Company

Vora P L, Farrell J E, Tietz J D and Brainard D H 1997b Digital colour cameras - 2 - spectral response. Technical Report HP-97-54, Hewlett-Packard Company

Vora P L, Farrell J E, Tietz J D and Brainard D H 1997c Linear models for digital cameras. In: Proc. 1997 IS\&T 5th Annual Conference, p. 377-382 